\title{
Repository structures for evolving federated database schemas
}

\author{
M. A. Jeusfeld ${ }^{*}$, M. Jarke+ \\ * Hong Kong University of Science \& Technology, ISMT, \\ jeusfeld@bi.ust.hk, Fax+851-2358-2421 \\ + Aachen University of Technology, 52056 Aachen, Germany, \\ jarke@informatik.rwth-aachen.de, Fax +49-241-8888-321
}

\begin{abstract}
Enterprise integration requires interoperability between processes and exchange between information systems. Both are heterogeneous, based on different vocabularies, and subject to continuous evolution. In this paper, we propose a method for managing the evolution of information exchange. Since a global unified model of enterprise information appears impractical, a market paradigm is developed where suppliers and consumers of information are matched. Technically, this market is established by ConceptBase, a system which manages meta data about the planned information exchanges. Since the meta data are explicit they are subject to querying and integrity checking. The unlimited meta class hierarchy of ConceptBase allows to represent and relate multiple views of the information exchange, especially the requirements view and the implementation view. The approach was applied in an interdisciplinary project developing a distributed quality management system.
\end{abstract}

Keywords

meta data, repository, deduction, systems analysis, quality management, federated database 


\section{INTRODUCTION}

As enterprises split up into smaller units, and as these smaller units start cooperating with each other even across company borders, the amount of information passed between independent units significantly increases. The desired lack of central management is accompanied by diverging schema definitions. Any local schema evolution potentially threatens the consistency of all application programs of the federated database. We will assume that the number of participating units is rather small (not more than several dozens). This is a reasonable restriction since dedicated information systems hardly cover a larger number of units in a company. We further assume that each participating system has an SQL server as front end which is used as a uniform data exchange language for the implementation model.

While SQL servers make up the target infrastructure, they are not suitable for specifying evolution. Therefore, we present a method within which the participating experts themselves can cooperatively specify/evolve the requirements to the information exchange. The result is used to support the update of the federated database schema. The management of the updates is implemented via the meta database system ConceptBase [JGJ*95] whose predicative query language is capable of quantifying over requirements and schema elements as well as over their relationships. The approach has been validated in the project WibQuS. The project participants developed autonomous quality management tools. Their information exchange is cooperatively designed and implemented using ConceptBase as a 'trader' component.

\section{AN ENTERPRISE-WIDE INFORMATION MANAGEMENT PROJECT}

A real-world application of the approach has been undertaken in the WibQuS project [JJS93,Wibq95]. The goal of this project was to discover and enact information flow between half a dozen of quality management methods covering the so-called quality cycle [Ima86,Pfei93]. Practically any department of an enterprise is directly or indirectly involved in quality management.

The experts working with the above tools share one common goal: the overall success of quality management. The achievement is highly dependent on the quality of information exchange between the experts. The central issue of this paper is to support quick evolution of the infrastructure for information exchange at an abstraction level suitable for experts unfamiliar with details of database systems. Integration of the participating quality management tools is an on-going, never-ending task for the quality team. Thus, a method for evolution that empowers the managers of the individual tools is critically needed.

The meta database is included together with its management system ConceptBase as one of the distributed systems (see node QualityTrader in fig. 1). Like all other systems it has an SQL interface to the network, i.e., it accepts SQL calls and can submit SQL calls to other systems. The participating systems use rather different software and hardware platforms, only agreeing on Internet and SQL 
standards. The QualityTrader can initialize the schema of new tools 'on the fly' by extracting the SQL code from the meta database (not further elaborated in this paper).

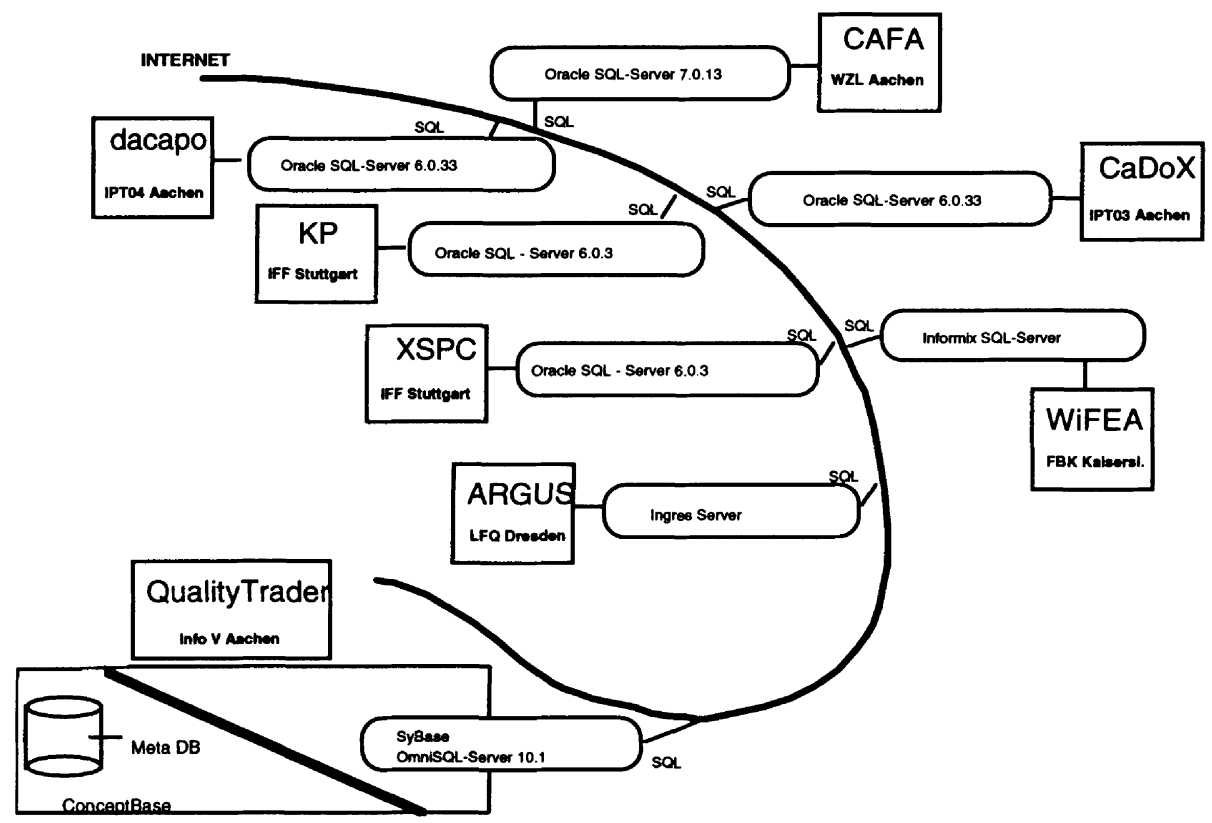

Figure 1: Federated database with trader component in WibQuS.

\section{ABSTRACTIONS OF FEDERATED DATABASES}

We will use a derivative of the knowledge representation language Telos [MBJK90] to design the schema of the meta database, especially integrity constraints that support the distributed evolution of the federated database schemas. This derivative, O-Telos [JGJ*95], is optimized for integrating metalevel information stemming from heterogeneous requirements and implementation languages.

Base predicates. O-Telos is a predicative language built on three base predicates:

( $\mathrm{x}$ in $\mathrm{c}$ ) expresses that the item $\mathrm{x}$ is an instance of the item $\mathrm{c}$,

(c isA d) declares $c$ as subclass of $d$, and

$(\mathrm{x} \mathrm{m} / \mathrm{n} \mathrm{y}) \quad$ states an attribute relation named $\mathrm{n}$ with category $\mathrm{m}$ between $\mathrm{x}$ and $\mathrm{y}$.

The arguments $\mathrm{x}, \mathrm{y}, \mathrm{c}, \mathrm{d}$ are names of objects or classes. All three predicates are in infix notation, the first two are binary predicates, the third is a 4-ary predicate. Besides these base facts, one can define deductive rules and integrity constraints. 
Rules and integrity constraints. O-Telos uses this facility to define built-in axioms like class membership inheritance and typing. For example, class membership inheritance is encoded by the deductive rule

$$
\forall \mathrm{x}, \mathrm{c}, \mathrm{d}(\mathrm{x} \text { in } \mathrm{c}) \wedge(\mathrm{c} \text { is } \mathrm{A} \mathrm{d}) \Rightarrow(\mathrm{x} \text { in } \mathrm{d})
$$

Note that this rule quantifies over classes. We call such formulas meta formulas. Besides the three base predicates we allow equality/inequality predicates. When the name ' $n$ ' of the attribute is irrelevant, we use the short form ( $\mathrm{x} \mathrm{m} \mathrm{y})$ instead of $(\mathrm{x} \mathrm{m} / \mathrm{n}$ y) defined by the deductive rule

$$
\forall \mathrm{x}, \mathrm{m}, \mathrm{n}, \mathrm{y}(\mathrm{x} \mathrm{m} / \mathrm{n} \mathrm{y}) \Rightarrow(\mathrm{xm} \mathrm{y})
$$

Application-specific rules and integrity constraints are not distinguished from the built-in axioms. They just have more constants referring to application classes like 'Employee' etc. ConceptBase provides an implementation for the above logical structures: the base predicates and the logical formulas are stored in a persistent object manager. Meta formulas are partially evaluated against the current extension of the base predicates to yield efficient representations.

These technical preliminaries allow us to define pre-fabricate properties of data models used below without explicitly assigning them to the data model. Instead, the designers can activate them on demand depending on the phase of the development process. For example, when the requirements to the information exchange are collected it makes no sense to demand that each request for information is assigned to a resource. However, when a request is to be implemented by a (SQL) query, then we may well require that the assignment is known. In the following, we motivate a four-layered classification [ISO90] of the models used in this paper.

A database schema can be regarded as a set of (logical) statements about admissible databases. Analogously, a data modeling language is a set of statements about admissible database schemas. Practically all data modeling languages use graphical representations. They differ in the set of node/link categories and the set of statements associated with them. We exploit this observation by setting up an abstraction hierarchy which considers the requirements on information exchange and the federated DB schema as descendants of the same so-called language model (fig. 2). The language model provides the syntactic and semantic means for representing dependencies between both models. These dependencies are used to map updates to the requirements on information exchange into an evolution of the federated DB schema.

The evolution method grounds on four abstraction levels for information known from repository systems [JJR90,ISO90]. The most abstract one is the language model (sec. 3.1) used to define both the implementation language (section 3.2) of SQL databases and the requirements language (section 3.3). The third abstraction level holds the federated DB schema and the requirements of information exchange. Evolution is specified as updates to the requirements and implemented on the federated database schema. 


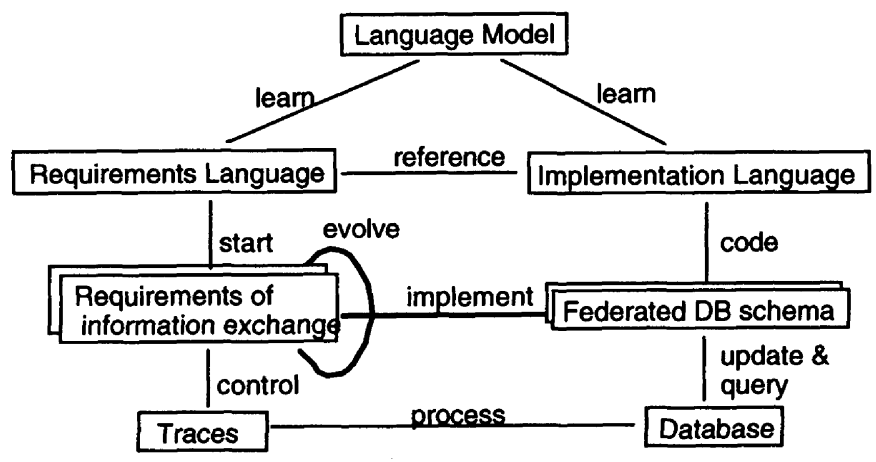

Figure 2: Levels of abstraction and their interconnection.

\subsection{The Language Model}

Evolution of federated database schema should be regarded as implementing the result of a negotiation between experts [AB89]. The degree of support depends on the formalization of such negotiation, i.e., a language. The model shown in fig. 3 is just a graphical representation of propositions ( $x$ in Object), ( $x$ references/references $y$ ), ( $t$ in Tool), (rq in Request), ( $r$ in Resource), ( $t$ references/issues $\mathrm{rq}$ ), ( $\mathrm{t}$ references/offers $\mathrm{rs}$ ), and ( $\mathrm{rq}$ references/accesses $\mathrm{rs}$ ). Formally, the language model is class level for the models in the subsequent subsections'.

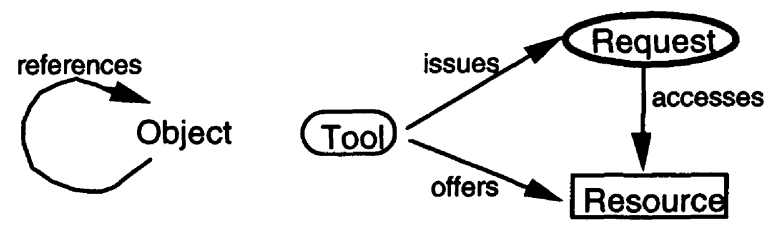

Figure 3: Language model (meta model) for offering resources and issuing requests.

\subsection{The Implementation Language SQL}

Figure 4 is the formal representation of how SQL is used as medium for information exchange: The SQL server requests information by SQL queries, it offers uniformly information as relations. SQL queries join 'from' relations and express membership by a 'where' clause. SQL queries may have parameters which are filled in at query time. Such queries are typically found in application

\footnotetext{
${ }^{1}$ Compare node layouts for identifying the corresponding classes.
} 
programs. Relations have attributes, some of them being primary keys. Relations have a (local) name within an SQL server. They can be made absolute by pre-pending the database name of the SQL server.

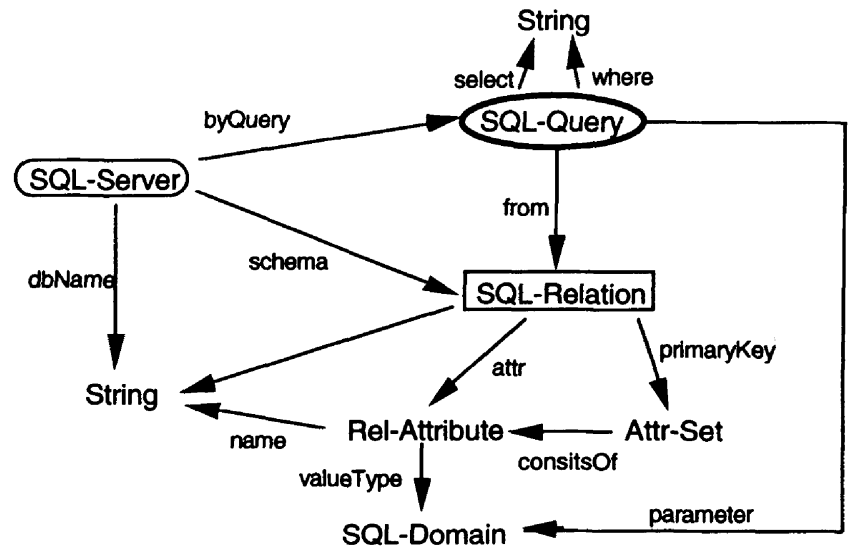

Figure 4: Abstract model of SQL servers as implementation platforms.

Semantic integrity of federated DB schemas can be expressed by first-order formulas in O-Telos. For example, the following axiom ensures uniqueness of relation names within the same SQL server. Note that the objects of the implementation model are now used as classes. Thus, the variable ' $r$ ' stands for an instance of an instance of the class 'Resource'.

$$
\begin{aligned}
\forall \mathrm{r}, \mathrm{s}, \mathrm{n}, \mathrm{srv} & ((\mathrm{r} \text { in } \mathrm{SQL}-\text { Relation }) \wedge(\mathrm{s} \text { in } \mathrm{SQL}-\text { Relation }) \wedge \\
& (\mathrm{n} \text { in String }) \wedge(\text { srv in } \mathrm{SQL}-\text { Server }) \wedge \\
& (\mathrm{r} \text { name } \mathrm{n}) \wedge(\text { s name } \mathrm{n}) \wedge \\
& (\text { srv schema } \mathrm{r}) \wedge(\text { srv schema } \mathrm{s})) \\
& \Rightarrow(\mathrm{r}=\mathrm{s})
\end{aligned}
$$

The abstract model of an SQL server is the framework for describing an implementation of a federated database. The implementation is indeed completely represented as instances of the classes in fig. 4.

\subsection{The Requirements Language and its Implementation}

The counterpart of the implementation language is the requirements language for federated databases - again an instance of the language model. The tool 'Participant' is used to classify the part systems of the distributed environment. They can appear in the role of information producers and consumers. 
The central task of the requirements language is to specify the match between the supply of information and the demand. Information sources can be decomposed into smaller parts. Sometimes a consumer will be interested in parts rather than the full information.

The three kinds of implementation links relate the requirements language to the implementation language: information sinks can be implemented by SQL queries, information sources are implemented as SQL relations.

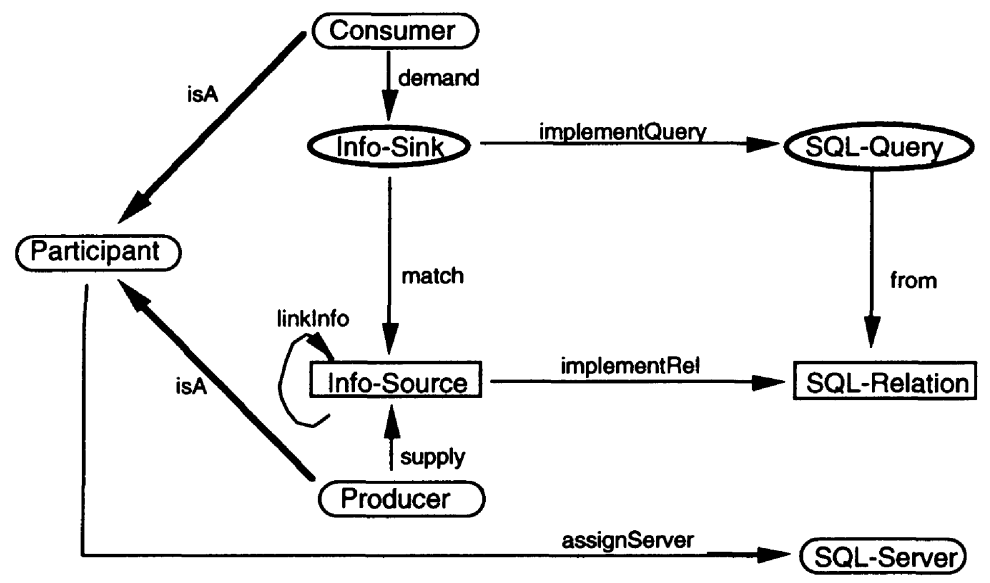

Figure 5: Requirements language for information exchange.

The 'linkInfo' reference between information sources is included in the model to state arbitrary references between information sources. Special cases of 'linkTo' like 'part-of' and 'subclass' can easily be included when the requirements language is mutated to a design language.

\section{EVOLUTION BY UPDATES TO REQUIREMENTS}

The models presented in the previous section constitute a set of ground facts of a meta database. The requirements specification is a set of facts of this meta database, more specifically, a collection of instances of the requirements language. Thus, evolution of the requirements is an update to the collection these instances. The development of a new federated database system is just a special case of evolution starting from an empty requirement specification.

We propose to regard the meta database as part of the distributed system (see fig. 1). Then, updates to the federated schemas can be executed at run-time. ConceptBase provides services for consistently updating and querying a meta database. 


\subsection{Policy for Evolution}

The requirements specification and the federated DB schema are both stored as objects in a meta database. Since evolution is represented by updates to the meta database, integrity constraints are a suitable choice for specifying the legal updates. Additional tests can be expressed by queries. All such integrity constraints and queries make up a policy for evolution. Since the evolution concerns the group of system designers, we argue that the group itself should be able to define and adapt the policy. Support can be provided by pre-fabricated meta formulas. They are formulated in terms of the language model. As an example, we show the 'mandatory' category. It classifies attributes of objects with at least one filler.

$$
\begin{aligned}
& \text { (Object references / mandatory Object) } \\
& \forall \mathrm{c}, \mathrm{m}, \mathrm{d}, \mathrm{x}(\mathrm{c} \text { mandatory } / \mathrm{m} \mathrm{d}) \Rightarrow((\mathrm{x} \text { in } \mathrm{c}) \Rightarrow \exists \mathrm{y}(\mathrm{y} \text { in } \mathrm{d}) \wedge(\mathrm{x} \mathrm{m} \mathrm{y}))
\end{aligned}
$$

The ground fact declares the 'mandatory' category as at the language model level. The quantified formula specifies what is understood by mandatory attributes. Note that this formula does not contain any constant referring to an object in the requirements or implementation language. It quantifies over those objects by variables 'c,m,d'. The variables ' $x, y$ ' quantify over instances of 'c' and 'd', i.e., over objects in the third level in the abstraction hierarchy (fig. 2). Assume the group of system designers wants that the 'demand' and 'supply' attributes of the requirements language are mandatory. This is done by inserting just two facts

(Consumer mandatory/demand Info-Sink)

(Producer mandatory/supply Info-Source)

into the meta database. This renders two specialized versions of the 'mandatory' constraint:

$$
\begin{aligned}
& \forall \mathrm{x}(\mathrm{x} \text { in Consumer }) \Rightarrow \exists \mathrm{y}(\mathrm{y} \text { in Info }- \text { Sink }) \wedge(\mathrm{x} \text { demand } \mathrm{y}) \\
& \forall \mathrm{x}(\mathrm{x} \text { in Producer }) \Rightarrow \exists \mathrm{y}(\mathrm{y} \text { in Info }- \text { Source }) \wedge(\mathrm{x} \text { supply } \mathrm{y})
\end{aligned}
$$

ConceptBase includes an incremental formula compiler [JJ91] which automatically generates the specialized versions. If a fact like '(Consumer mandatory/demand Info-Sink)' is removed from the meta database then the corresponding constraint will also be removed. Thereby, change of policy is technically very easy. Categories like 'single' (at most one filler allowed for an attribute), 'invsingle' (at most one object referring to the same attribute value) etc. can be defined in the same way. ConceptBase even allows definition on the fly, i.e.., when partial models are already in its meta database without compromising on consistency.

The presence of these statements in the meta database enforces the semantics expressed by the formulas associated to them. Note that there are periods of time during the evolution of the requirements specification when some/all of the statements are not desirable, e.g., the association of 
an information source to a producer may become into effect only at the end of a long transaction (on the meta database) because there are multiple choices. The benefit of the pre-fabricated categories is that they can easily be 'switched' on and off by the system designers without ever having to change (or even understand) the predicative formulas that define them.

Before demanding the integrity one may use queries to check to what degree a given requirement specification and/or federated database schema fulfills the conditions. For example, the query

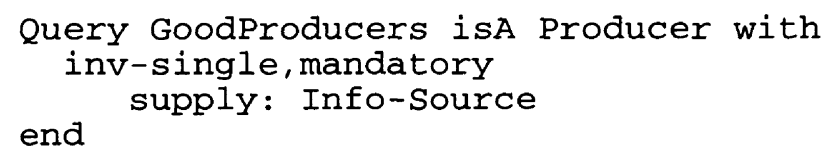

and its relative complement 'NOT-GoodProducers' partitions the producers into those who uniquely supply at least one information source and those who don't. Not incidentally, the categories 'invsingle' and 'mandatory' do the job.

\subsection{Implementation of Evolution}

The requirements specification is not complete in the sense that a federated database schema can be automatically generated from it. The information sources are aggregations of the relations implemented by them. Details about the attributes of the relations are not represented as properties of the information sources. On the other side of the requirements language, information sinks are even more abstract than there counterparts on the SQL side, namely queries and views. Nevertheless, a formal representation of interrelationships between the two languages yields some effective support in the evolution task. We briefly mention the kind of services ConceptBase offers for the implementation mapping.

The relations to be managed by a certain SQL server can be derived from the assignment of a 'supplier' tool to the SQL server: each relation implementing an information source offered by the supplier is to be managed by the SQL server in question, or as an O-Telos rule

$$
\begin{gathered}
\forall \mathrm{p}, \mathrm{i}, \mathrm{s}(\mathrm{p} \text { supply } \mathrm{i}) \wedge(\mathrm{p} \text { assignServer } \mathrm{s}) \wedge(\mathrm{i} \text { implementRel } \mathrm{r}) \\
\Rightarrow(\mathrm{s} \text { schema } \mathrm{r})
\end{gathered}
$$

The SQL relations and queries affected by a removal of an information source (or request) can be effectively computed by following the 'implementRel' links in the meta database. For a query, the participating relations can be determined by first extracting the matched information sources and then accessing the relations implementing them. This constitutes already the 'from' part of the SQL query.

All services in this section are described by the means of deductive rules which check the meta database and extract derived information useful for the services. Integrity constraints are, with a few exceptions, expressed by assigning attributes of the requirements and implementation language to 
pre-fabricated categories. Thereby, designers not familiar with predicate calculus can easily set up the terms under which evolution can take place. All rules and integrity constraints are effectively computable by interpreters. The effect of an incremental update can be computed by evaluating partially instantiated versions of certain rules.

To illustrate the use of the above techniques we turn back to the example of quality management. Figure 6 contains a snapshot of a certain stage in the requirements specification for WibQuS. A new information sink 'Product-Description' has been inserted as demand for the participant WiFEA. This demand is not matched by any information source, i.e. it is an answer of the query 'ResolvedSinks-'.

The designers may find out that the requested information must be accessed from a CAD database not yet included in the architecture. It is included by inserting the following facts into the meta database and matched to the unresolved request. The product structure is linked to the product properties managed by the Dacapo system.

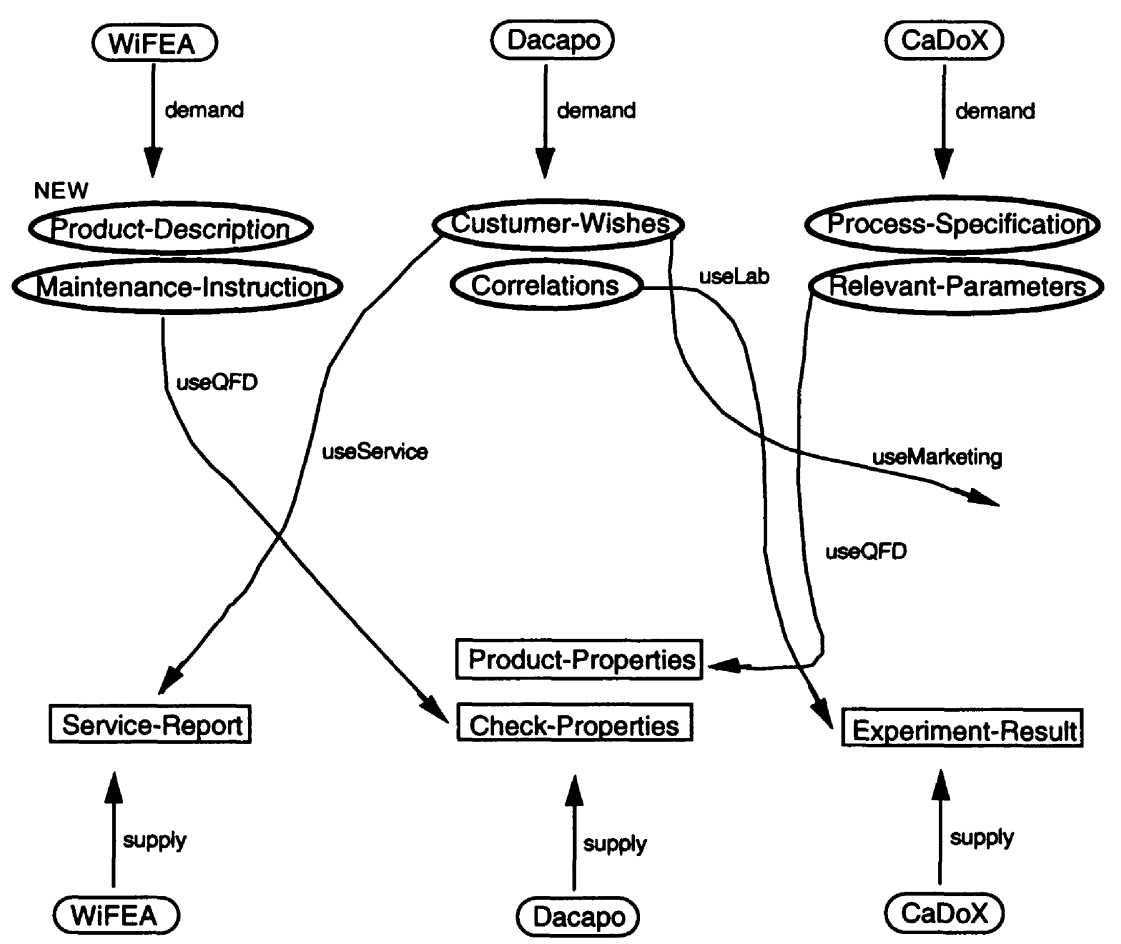

Figure 6: Requirements specification in the WibQuS case (excerpted from [Wibq95]). 


\section{DISCUSSION}

Kashyap and Seth [KS94] proposed an architecture with information providers, consumers, and brokers. The task of the broker (trader) is to transform a consumer query into a query on the providers databases by comparing semantic and structural similarities. It is an approach for information access in federated databases where the part schemas have evolved independently. Our proposal is tightly-coupled [SL90]: the group of designers having a common goal collaboratively define the requirements of information exchange (interschema knowledge).

Negotiation in federated databases was proposed by Alonso and Barbera [AB89]. Their main concern is to estimate the price for accessing information in order to organize the 'information market place'. They also propose to materialize views of often-used queries on local sites. Such views are regarded as crucial for query optimization in distributed databases (see [RES93]).

The institution of an active trader has an important role in Open Distributed Processing (ODP) [PM93]. The properties of a service and its arguments (opposed to schema information in the federated database case) are defined in algebraic terms. The trader has the task to compute a match between a service request and a service offer. Type conversions of arguments are performed for both service request and answer transmission.

Recent work on schema evolution concentrates on object-oriented databases. Compatibility with existing applications is achieved by either versioning of objects and schemas [Clam94] or by objectoriented views [TS93].

We proposed a framework for the evolution of a federated DB schema via updates to a meta database. The meta database distinguishes between requirements of information exchange and their implementation in SQL databases. Evolution of information requests (queries) is supported concurrently by representing their match to information resources.

The group of DB designers can set-up an evolution policy by enforcing suitable integrity constraints on the meta database. An extensible set of predefined categories frees the designers from the task of encoding predicate calculus. The implementation of the coarse-grained updates to the requirements specification to an evolution of the federated DB schema exploits the power of a deductive query language. Services include re-assignment of information resources and assistance on the code generation for SQL queries.

A real-life application has been undertaken in the WibQuS project. The meta database is managed at the production phase of the distributed system by the ConceptBase system. The meta database keeps being accessible and updatable at any time. The approach has proven to work in an environment of heterogeneous SQL servers running on different hardware platforms. The constraint and query facilities were especially productive. In the early phase only few constraints were enforced. As experts became more familiar with the use of ConceptBase and the approach, more and more constraints were activated.

Extensions are possible on both the requirements and the implementation language, e.g., by mapping to an object-oriented database instead of relational SQL. The ability to represent multiple views of the federated system within one meta database is the most important strength of ConceptBase. The semantic categories defined by meta formulas make these views manageable for 
the development team.

Acknowledgments. This work was supported in part by the German Ministry of Research and Technology under grant 02QF3004/1, and by the European Community under ESPRIT Basic Research Action 6810 (Compulog 2) and Working Group No. 8319 (ModelAge). We would like to thank Rainer Gallersdörfer, Peter Peters, and Peter Szczurko for the fruitful collaboration in WibQuS for almost three years. A big thank goes to the members of the ConceptBase team, esp. Martin Staudt, Kai von Thadden and René Soiron, for investing much of their spare time in supporting the WibQuS project, and to our student programmers, Almudena Rodriguez-Pardo, Markus Mandelbaum and Reiner Nix.

\section{REFERENCES}

[AB89] Alonso, R., Barbará, D. (1989). Negotiating data access in federated database systems. 5th Intl. Conf. Data Engineering, pp. 56-65, 1989.

[Aka90] Akao, Y. (1990). Quality function deployment - integrating customer requirements into product design. Productivity Press, Cambridge, 1990.

[Clam94] Clamen,S. (1994). Schema Evolution and Integration. Distributed and Parallel Databases, 2, 1, pp. 101-126, Jan. 1994.

[Ima86] Imai, M. (1986). Kaizen - The Key to Japan's Competitive Success. MacGraw-Hill, New York, 1986.

[ISO90] ISO/IEC 10027 (1990). Information technology - information resource dictionary system (IRDS) - framework. ISO/IEC International Standard, 1990.

[JGJ*95] Jarke, M., Gallersdörfer, R., Jeusfeld, M.A., Staudt, M., Eherer, S. (1995). ConceptBase - a deductive object base for meta data management. Journal of Intelligent Information Systems, 4, 2, pp. 167-192, 1995.

[JJ91] Jeusfeld, M.A., Jarke, M. (1991). From relational to object-oriented integrity simplification. Proc. 2nd Intl. Conf. Deductive and Object-Oriented Databases, LNCS 566, Springer-Verlag, pp. 460-477, 1991.

[JJR90] Jarke, M., Jeusfeld, M.A., Rose, T. (1989). A software process data model for knowledge engineering in information systems. Information Systems, 15, 1, pp. 85-116, 1990.

[JJS93] Jarke, M., Jeusfeld, M.A., Szczurko, P. (1993). Three aspects of intelligent cooperation in the quality cycle. Intl. J. of Intelligent and Cooperative Information Systems, 2, 4, pp. 355-374, 1993.

[MBJK90] Mylopoulos, J., Borgida, A., Jarke, M., Koubarakis, M. (1990). Telos: a language for representing knowledge about information systems. ACM Trans. Inf. Syst., 8, 4, pp. 325$362,1990$.

[Pfei93] Pfeifer, T. (1993). Qualitätsmanagement.. Carl-Hanser-Verlag, Germany, 1993.

[PM93] C. Popien, B. Meyer, Federating ODP traders: an X.500 approach. Proc. of IEEE Intl. Conf. on Communications (ICC), Geneva, pp. 313-317, May 1993. 
[RES93] Roussopoulos,N., Economou,N., Stamenas,A. (1993). ADMS: a testbed for incremental access methods. IEEE Trans. Knowledge and Data Engineering, 5,5, pp. 762-774, Oct. 1993.

[SL90] Sheth,A.P., Larson,J.A. (1990). Federated database systems for managing distributed, heterogeneous, and autonomous databases. ACM Computing Surveys, 22, 3, pp. 183-236, Sept. 1990.

[SNJ94] Staudt,M., Nissen, H.W., Jeusfeld, M.A. (1990). Query by Class, Rule, and Concept. Journal of Applied Intelligence, 4, pp. 133-156, 1994.

[TS93] Tresch, M., Scholl M.H. (1993). Schema transformation without database reorganization. SIGMOD Record, 22, 1, pp. 21-27, 1993.

[Wibq95] WibQuS Team (1995). Protocols of general meetings. Unpublished memo, WZL-MTQ Aachen, 1995.

\section{BIOGRAPHY}

Manfred Jeusfeld is visiting assistant professor at the information systems group of the Hong Kong University of Science and Technology, on leave from Aachen University of Technology. He has worked on amalgamation of deductive and object-oriented data models and on the design of distributed information systems. He is the principal designer of ConceptBase, a multi-user meta database manager.

Matthias Jarke is professor of information systems and chairman of the computer science department at Aachen University of Technology, Germany. His research addresses information systems support for business, design and engineering applications focusing on distributed cooperative work processes. He has served as project coordinator for two European ESPRIT projects in this area, DAIDA and NATURE. He is Editor-in-Chief of the journal Information Systems and Vice President Advanced Technologies of the International Association for Information Systems. 